\title{
On the progressive nature of grain crushing
}

\author{
Matteo O. Ciantia ${ }^{1 *}$, Gema Piñero ${ }^{2}$, Jian Zhu $^{1}$ and Tom Shire ${ }^{1}$ \\ ${ }^{1}$ Dept. Civil and Environmental Engineering, Geotechnics Section, Imperial College London, UK \\ ${ }^{2}$ Institute of Telecommunications and Multimedia Applications, Universitat Politecnica de Valencia, Spain
}

\begin{abstract}
In this work acoustic emission (AE) is used as experimental evidence of the progressive nature of grain crushing. Stress controlled high pressure oedometric compression test are carried out on $1.2 \mathrm{~mm}$ monodisperse samples of glass beads. It was observed that the granular assembly starts to experience particle breakage at a vertical stress of about $25 \mathrm{MPa}$. When this yield pressure is exceeded the glass beads start to break emitting loud impulsive sound and the vertical displacement increases rapidly. The load was increased beyond the yield stress and at each increment while the vertical stress remained constant the sample continued to emit sound. The emission of sound at a constant vertical stress indicates that crushing is a progressive failure mechanism; once the first crushing event occurs, the structure starts to rearrange causing other crushing events to occur and additional settlement. In particular, two signal processing algorithms are used on the samples of the acoustic signal to obtain two additional metrics of the crushing evolution. The first is the cumulative energy versus time. The second is the number of crushing events versus time, which is based on the automatic detection of the peaks of the sound signal envelope. There is a clear correlation between the cumulative acoustic energy emitted and the observed sample displacement. Using laser scanning, the evolution of the particle size distribution and particle shape are measured in detail so that a link between the acoustic data and the crushing intensity is established. The crushing intensity was controlled using materials with different strengths.
\end{abstract}

\section{Introduction}

It is well acknowledged that most solids emit low-level seismic signals when they are deformed or stressed [1]. Such phenomenon of generating signals is termed as acoustic emission (AE) activity. AE are the mechanically induced elastic waves typically generated by grain rearrangement, grain inter-friction and crack formation for granular geomaterials [2]. The measurement and quantification of $\mathrm{AE}$ was initially developed for the attempt to predict the rock bursts for mining industry, and recently increasingly utilized for laboratory-scale studies for granular materials given their discrete nature $[3,4]$. Since the measurements of macroscopic stress and total deformation do not allow the grain-scale inspections of the microscopic responses of the particles, the application of acoustic emission techniques provides a new approach to looking inside the aggregates when they are stressed. As emphasized by Hardy Jr [1], although AE does not directly determine the basic mechanical parameters like stress and strain, it does provide a complement for the mechanical measurements of stress and displacement so that the interactions among particles can be revealed. Hidalgo et al. [5] used acoustic emission to study the force chain evolutions of granular materials with a $140 \mathrm{~mm}$ diameter cylinder sample while smaller sized specimens were also used to study the particle size distribution evolutions of powders [6]. Frequency and attenuation of measured acoustic events can be related to certain physical and mechanical processes of granular materials under shearing or compression. Measurement of AE are realized by detecting and monitoring the generation and the propagation of elastic waves due to the release of stored elastic strain energy during material deformation and abrupt grain interaction. The total energy of the AE is dominated by its sources, and usually described in terms of attenuation and signal pulse frequency [2].

Grain crushing is an elastic strain energy release mechanism that produces $\mathrm{AE}$ with a loudness and frequency within the range of audible sound. It is hence possible to detect and record crushing events within a stressed geomaterial using normal microphones. Whereas to capture other source mechanisms of AE such as grain friction and rearrangement more advanced sensors are required.

Experimental [7] and numerical [8] studies have shown how grain crushing in granular media is a progressive type of mechanism. Upon a small load increment, if a grain crushes, its load must be redistributed to the remaining intact grains. This load redistribution may lead to secondary failures of elements and triggers avalanches of failures before the load is distributed to a sufficient number of intact elements to return to an equilibrated state [2].

\footnotetext{
${ }^{*}$ Corresponding author: m.ciantia@imperial.ac.uk
} 
Due to the discrete nature of acoustic emissions associated with failure of individual grains, the progressive material degradation should also cause avalanches of AE. The focus of this paper is to hence experimentally demonstrate how the progressive nature of crushing in granular materials can be indirectly observed through the recording and analysis of AE. High pressure one-dimensional compression tests were performed. In addition to stresses and strains, size and shape evolution was also monitored. To control the level of crushability, the tests were run on mixtures of crushable and non-crushable spherical particles. This was complimented by AE analysis of the sound emitted during compression. A simple methodology to estimate the number of crushing events occurring within the sample is also proposed.

\section{Testing methodology}

\subsection{Materials tested}

Spherical glass and steel beads were tested. The physical properties of the materials are given in Table 1. Sphericity (perimeter of equivalent circle $\div$ real perimeter) and aspect ratio (ratio of Ferer min and max diameters) were determined from the QicPic laser image analysis instrument [9]. Both materials are very spherical and have uniform particle size distributions (low $\mathrm{R}_{\mathrm{D}}$ values, where $\left.\mathrm{R}_{\mathrm{D}}=\mathrm{D}_{90} / \mathrm{D}_{10}\right)$. The glass beads have a slightly larger median diameter. The steel beads are uncrushable under the stresses applied here.

Table 1 Properties of granular materials tested

\begin{tabular}{lcc}
\multicolumn{1}{c}{ Property } & Glass Beads & Steel Beads \\
\hline Density (-) & 2.60 & 7.81 \\
Shear Modulus (GPa) & 70 & 200 \\
Nominal Strength (MPa) & 70 & 1000 \\
Median diameter, $\mathrm{D}_{50}(\mathrm{~mm})$ & 1.29 & 1.0 \\
Relative distribution, $\mathrm{R}_{\mathrm{D}}(-)$ & 1.13 & 1.02 \\
Sphericity, $\mathrm{S}^{\mathrm{PQ}}(-)$ & $0.93-0.95$ & $0.93-0.95$ \\
Aspect ratio, $\mathrm{A}_{\mathrm{r}}(-)$ & $0.96-0.99$ & $0.98-0.99$ \\
\hline
\end{tabular}
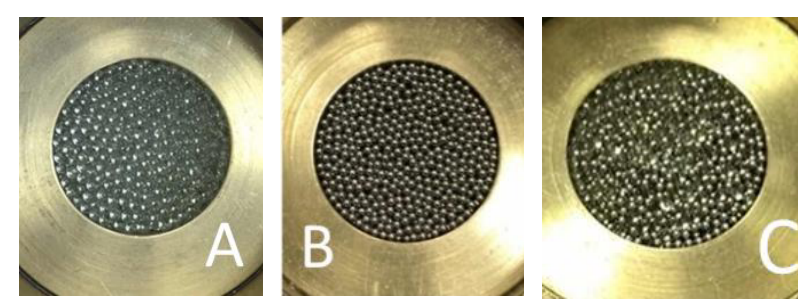

Fig. 1. Picture of the glass $(\mathrm{G})$, steel $(\mathrm{S})$, and mixture $(\mathrm{M})$ samples in the oedometric cell.

In the following, results from tests on three samples are presented: (i) glass beads only (G), (ii) steel beads only (S), (iii) $50 \%$ glass and $50 \%$ steel by volume (M) and in Fig. 1 photographs of the three samples before the high pressure oedometric compression test are reported.

\subsection{One-dimensional compression}

A load-controlled oedometer apparatus was used to carry out the one-dimensional compression tests. A $20 \mathrm{~mm}$ diameter confining ring was used to enclose the bead mixtures. Vertical loads were applied in stages using dead-weights, up to a maximum vertical pressure of 49.7 $\mathrm{MPa}$. Samples were prepared using a combination of pouring and light tapping. The mixed sample was thoroughly mixed prior to placement to minimise particle segregation. The initial void ratios are given in Table 2 . Displacement was monitored throughout the loading stage. Each loading stage was maintained until the vertical deformation was less than $0.003 \mathrm{~mm} / \mathrm{s}$ for at least 20 minutes. Following unloading the particle size and shape characteristics of the glass fraction was determined using the QicPic instrument [9].

Table 2. Properties of granular mixtures.

\begin{tabular}{lccc} 
& Glass & Steel & Mixture \\
\hline Mass (gr) & 9.13 & 27.46 & 18.19 \\
Glass fraction (\% mass) & 100 & 0 & 33.35 \\
Glass fraction (\% vol) & 100 & 0 & 50 \\
Initial void ratio, e $0(-)$ & 0.593 & 0.59 & 0.603 \\
Final void ratio, $\mathrm{e}_{\mathrm{f}}(-)$ & 0.225 & 0.531 & 0.353 \\
Yield stress, $\sigma_{\mathrm{y}}(\mathrm{MPa})$ & 27 & - & 30 \\
\hline
\end{tabular}

\subsection{Acoustic emission analysis}

$\mathrm{AE}$ were recorded and analysed for each test using the embedded microphone of a MacBook Air and Audacity ${ }^{\circledR}$, a free open source digital audio recording and editor software (http://www.audacityteam.org/). The laptop was placed about $30 \mathrm{~cm}$ from the oedometer, the sampling frequency was $f_{s}=44100 \mathrm{~Hz}$, resulting in a sampling time of $\mathrm{T}_{\mathrm{s}}=1 / f_{s}=22.7 \mu \mathrm{s}$ and a signal bandwidth between 0 and $22050 \mathrm{~Hz}$ for the recorded sound. The sound signal of the $\mathrm{G}$ test is shown in Fig. 2a, the units are arbitrary. The maximum range permitted is between -1 and +1 . It can be appreciated that the signal has a lot of peaks, but also bursts of energy between the peaks. The detailed shape of peaks and bursts can be appreciated in Fig. $2 \mathrm{~b}$ where three bursts are captured. Their peak amplitudes are different, but their burst durations are quite similar. Every burst has a duration around $60 \mathrm{~ms}$. Two measures of acoustic emission were attained, namely sound cumulative energy $\left(E_{c u}\right)$ and the number of acoustic events $\left(N_{c}\right)$ during a loading increment. If $s(\mathrm{t})$ is the sound signal, the recorded signal can be described by its discrete-time signal $s(n)$ :

$$
s(n)=s\left(n T_{s}\right)=\left.s(t)\right|_{t=n T_{s}}, n=0,1 \ldots
$$

Therefore, the discrete-time cumulative energy is expressed as:

$$
E_{\text {cum }}(n)=\sum_{k=0}^{n}|s(k)|^{2}
$$




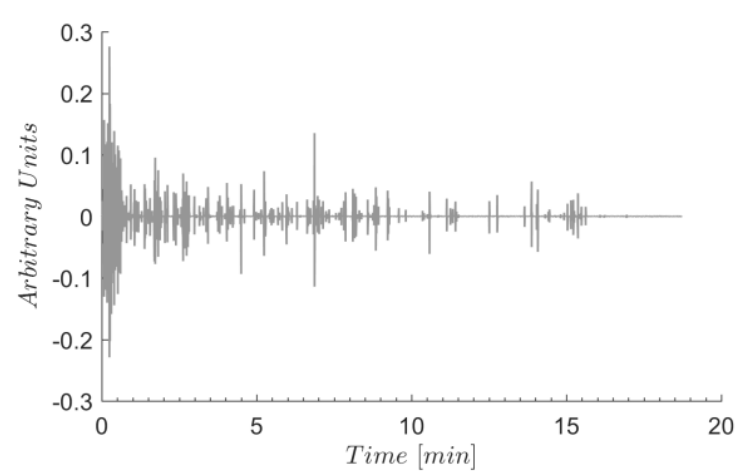

a)

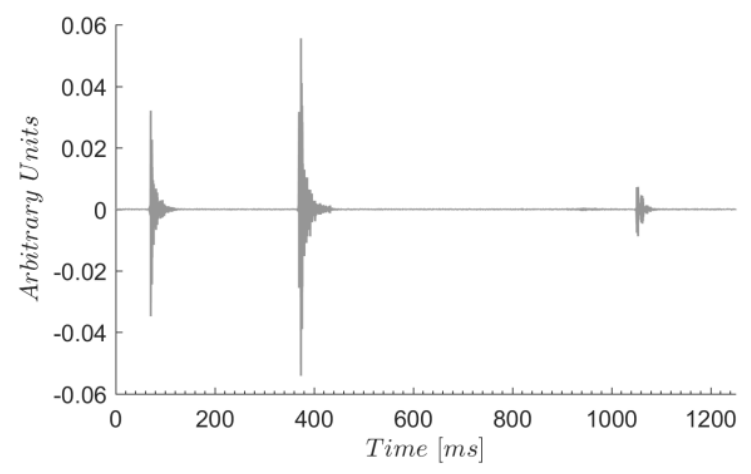

b)

Fig. 2. Sound signal for the G test; a) 12 minutes recording and b) $1200 \mathrm{~ms}$ sound signal segment detail showing three crush events.

To avoid unwanted low-frequency noise in lab influencing the calculation of $E_{c u}$, a high-pass-filtered (HPF) signal of cut-off frequency $f_{c}=8000 \mathrm{~Hz}$ and an attenuation of $60 \mathrm{~dB}$ in the stop band The resulting signal is called $s_{\mathrm{HP}}(\mathrm{n})$. In Fig. $2 \mathrm{~b}$ a sound segment of the original $\mathrm{AE}$ recording is shown where three bursts (each one associated with a crushing event) can be identified. It can be appreciated that the three follow a similar pattern but with different amplitudes. The high-pass filtered signal presents similar behaviour, and hence the crush events can be identified by a certain pattern (i.e. signal shape) although the amplitude may vary. Therefore, signal $\mathrm{s}_{\mathrm{HP}}(\mathrm{t})$ can be expressed as

$$
S_{H P}(t)=\sum_{k=1}^{N_{c}} A_{k} p\left(t-\tau_{k}\right)
$$

where $A_{k}$ is the amplitude of the kth repetition of the pattern $p(t), \tau_{k}$ is the time instant when $p(t)$ starts and $N_{c}$ is the number of crush events. We can rewrite the above equation in discrete-time

$$
S_{H P}(n)=\sum_{k=1}^{N_{c}} A_{k} p\left(n-n_{k}\right)
$$

Where $p(n)=\left.p(t)\right|_{t=n T_{s}}=p\left(n T_{s}\right)$, index $n_{k}=\left[\tau_{k} f_{s}\right]$ and [.] means the nearest integer to the argument. If we call $R_{p}(n)=E[p(k) p(k+n)]$ the autocorrelation function of the pattern, then $R_{p}(n)$ can be estimated as:

$$
R_{p}(n)=\frac{1}{N_{p}-|n|} \sum_{k=0}^{N_{p}-1-|n|} p(k) p(k+|n|),-N_{p}<n<N_{p}
$$

where $N_{p}$ is the length of $p(n)$ in number of samples. Therefore, the cross-correlation function of the discretetime signal $\mathrm{S}_{\mathrm{HP}}(n)$ and the pattern $p(n)$ is computed as

$$
x(n)=\sum_{k=1}^{N_{c}} A_{k} p\left(n-n_{k}\right) p(-n)=\sum_{k=1}^{N_{c}} A_{k} R_{p}\left(n-n_{k}\right)
$$

where $x(n)$ is a new signal that enhances the peaks of the original $\mathrm{S}_{\mathrm{HP}}(\mathrm{n})$ signal. At this point the amplitude envelope of $x(n)$ is computed by means of a Hilbert transform [11], and a crushing event is summed up to the count when a peak of the envelope is above a certain threshold. A parametric study assessing the influence of threshold on the Ncr-time curve was performed and for this type of AE signals, the method proposed resulted to be independent from the threshold value chosen.

\section{Results}

The compression curves for the three samples are shown in Fig. 3. It is clear how particle breakage induces large irreversible deformations which are proportional to the amount of glass beads in the sample. In Fig. 5 it is clear how the crushing induced deformation evolves with time, with rapid crushing on yield (point $A, \sigma_{z}=33 \mathrm{MPa}$ ), followed by significant further displacement over time. Upon a single stress increment of $5 \mathrm{MPa}$ a displacement of about $2 \mathrm{~mm}$ is recorded (Point B).

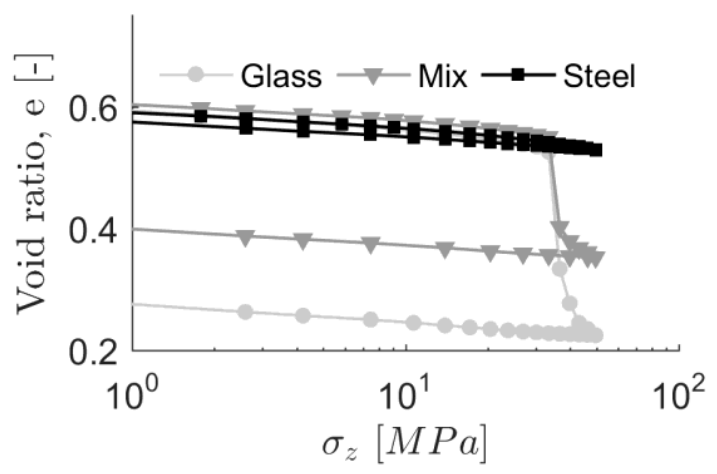

Fig. 3. Load unload oedometric compression; comparison of the void ratio evolution for the $\mathrm{G}, \mathrm{S}$ and $\mathrm{M}$ tests.

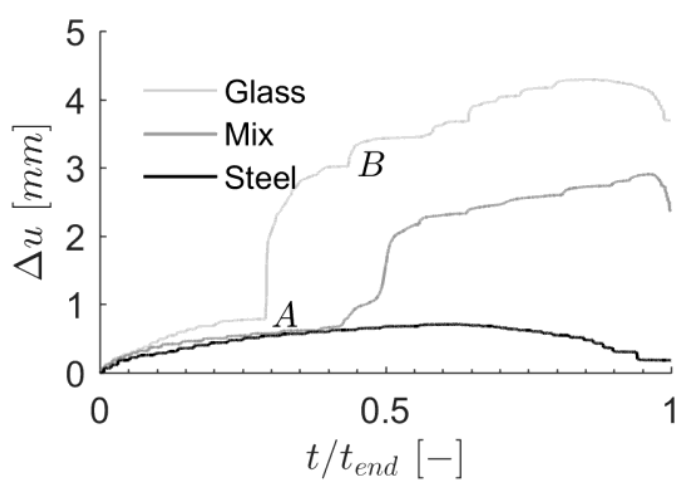

Fig. 4. Temporal evolution of displacements for the three tests. 
The progressive nature of the crush induced deformation is well reflected in the $\mathrm{AE}$ analyses in terms of both $E_{c u}$ and $N_{c r}$, as shown in Fig. 5 for the first yield of the glass sample G. This clearly demonstrates that AE analysis can capture the energy release during particle crushing. Following [9] QicPic laser image analyses can be performed on the sample at the end of the experiment and the particle size distribution (PSD) and sphericity evolution can be used as another indicator of crushing evolution (Fig. 6).

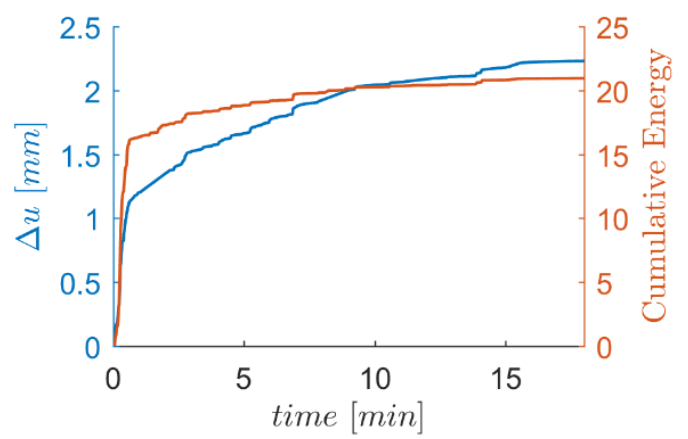

a)

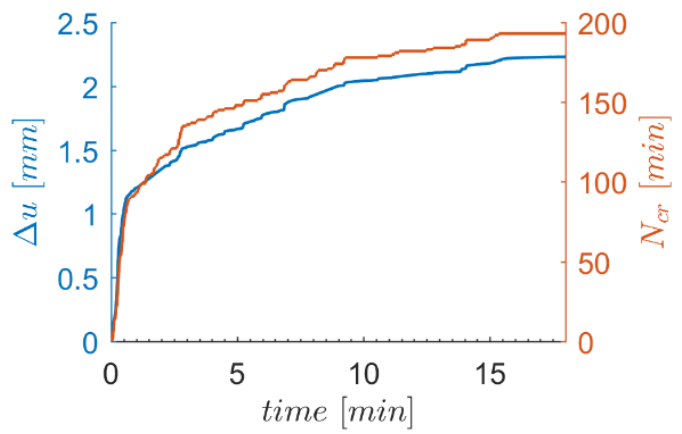

b)

Fig. 5. Comparison of temporal evolution of a) cumulative energy and b) number of crushing events for the of Glass sample test from point A to B in Fig. 4.

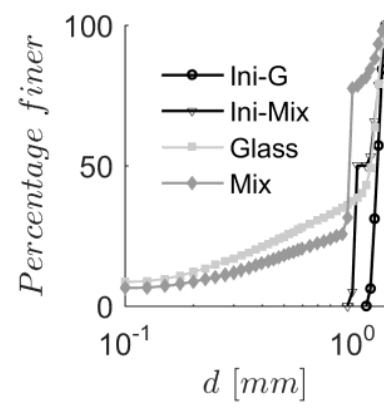

a)

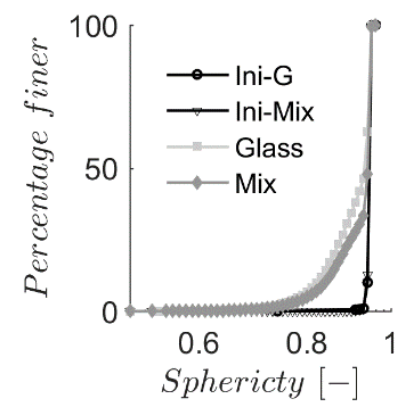

b)
Fig. 6. Initial and final a) PSD and b) sphericity measurements of the three samples tested.

\section{Conclusions}

In this paper two signal processing algorithms are used to obtain two additional metrics of crushing evolution. The first is the cumulative energy versus time, and the second is the number of crushing events versus time. This last measurement is based on the automatic detection of the peaks of the sound signal envelope. The strong correlation between the time displacement curves and the time $E_{c u}$ and $N_{c r}$ indirectly confirm the progressive nature of grain crushing. Upon a small load increment, if a grain crushes a sharp burst is emitted, the load carried by the element is redistributed to the remaining intact grains leading to secondary failures of elements triggering avalanches of crushing episodes. The determination of the number of crushing events is a novel measure that may be used as an extra variable for DEM crushing model validation.

\section{Acknowledgments}

This study was undertaken as part of an Imperial College London Junior Research Fellowship Research Grant.

\section{References}

1. Hardy JR, H.R. (2005). Acoustic emission microseismic activity: volume 1: principles, techniques and geotechnical applications. CRC Press

2. Michelmayr, G.; Cohen, D. and Or, D. (2012). Sources and characteristics of acoustic emissions from mechanically stressed geologic granular media-A review. Earth-Science Reviews, 112(3), 97-114.

3. Michlmayr, G., Cohen, D., \& Or, D. (2013). Shearinduced force fluctuations and acoustic emissions in granular material. Journal of Geophysical Research: Solid Earth, 118(12), 6086-6098.

4. Sha Luo S., Diambra A., Ibraim E. (2016) Application of acoustic emission on crushing monitoring of individual soil particles in uniaxial compression test. $32^{\text {nd }}$ European Conference on Acoustic Emission Testing Prague, Czech Republic, September 07-09, 2016

5. Hidalgo, R.C., Grosse, C.U., Kun, F., Reinhardt, H.W. \& Herrmann, H.J. (2002). Evolution of percolating force chains in compressed granular media. Physical Review Letters, 89(20), 205501-4.

6. Carson, G., Mulholland, A.J.; Nordon, A.; Tramontana, M.; Gachagan, A. and Hayward, G. (2008). Particle sizing using passive ultrasonic measurement of particle-wall impact vibrations. Journal of Sound and Vibration, 317(1), 142-157.

7. Tapias, M., Alonso, E. E., \& Gili, J. (2016). Compressibility, grain breakage and time-dependent behavior of gap-graded aggregates of sugar cubes. Soils and Foundations.

8. Ciantia, M. O. Arroyo, M., Calvetti, F. \& Gens, A. (2016) A numerical investigation of the incremental behaviour of crushable granular soils. Int. J. Numer. Anal. Meth. Geomech. 40(13) 1773-1798.

9. Cavarretta, I., Coop, M., \& O'Sullivan, C. (2010). The influence of particle characteristics on the behaviour of coarse grained soils. Géotechnique, 60(6), 413-423.

10. Rabiner, L.R., Gold, B. (1975) Theory and application of digital signal processing, ed. PrenticeHall, Englewood Cliffs, N.J. 